\title{
Regulation and Integral Control of an Underactuated Robotic System Using IDA-PBC with Dynamic Extension.
}

\author{
Y. R. Teo ${ }^{1}$, Student Member, IEEE, A. Donaire ${ }^{1}$, Member, IEEE and T. Perez ${ }^{1}$, Member, IEEE
}

\begin{abstract}
This paper proposes a method for design of a set-point regulation controller with integral action for an underactuated robotic system. The robot is described as a port-Hamiltonian system, and the control design is based on a coordinate transformation and a dynamic extension. Both the change of coordinates and the dynamic extension add extra degrees of freedom that facilitate the solution of the matching equation associated with interconnection and damping assignment passivity-based control designs (IDA-PBC). The stability of the controlled system is proved using the closed loop Hamiltonian as a Lyapunov candidate function. The performance of the proposed controller is shown in simulation.
\end{abstract}

\section{INTRODUCTION}

The trend in the manufacturing towards high-rate production and flexible configuration of production lines has lead to the use of advanced automation of light robotics in different industries. In many applications, the high-speed motion of the robot manipulator can excite the resonant modes of the reconfigurable base supporting the robot. This can result in unwanted vibration that degrades the accuracy of the robot and may compromise the quality of task. The base on which the robot is mounted is usually passive (no actuators are used to control the vibration of the base), and as a result the system that comprise of the robot and the base can be classified as an underactuated system-not every degree of freedom of the system can be actuated. Specifically, let the configuration variables of the mechanical system be denoted by $\mathbf{q} \in \mathcal{Q}$ and the conjugate momenta be $\mathbf{p} \triangleq \partial L / \partial \dot{\mathbf{q}} \in \mathcal{P}$, where $L$ is the Lagrangian. The system is called fully actuated if the forces, $\tau \in \mathcal{F}$, produced by actuator configuration is such that $\operatorname{dim} \mathcal{F}=\operatorname{dim} \mathcal{P}$. If $\operatorname{dim} \mathcal{F}<\operatorname{dim} \mathcal{P}$ the system is said to be underactuated [1]. In recent years, new control designs have been proposed for the class of underactuated systems (see e.g. [2], [3], [4], [5]).

Mechanical systems, as many engineering complex systems, can be written into port-Hamiltonian system (PHS) form [6]. An interesting feature of PHS is that variables and functions of the state-space representation have interpretation in terms of the physical phenomena of the system. An important control property of PHS is that of passivity. In the port-Hamiltonian $(\mathrm{PH})$ framework, these characteristics have been exploited to develop control techniques based on

\footnotetext{
${ }^{1}$ Y. R. Teo, A. Donaire, and T. Perez are with the School of Engineering and PRC CDSC, The University of Newcastle, 2308 Callaghan, NSW, Australia, emails: Yik.Teo@newcastle.edu.au, Alejandro. Donaire@newcastle.edu.au, Tristan.Perez@newcastle.edu.au
}

passivity and energy, for example control by interconnection, and interconnection and damping assignment passivity-based control [7], [8], [9]. The IDA-PBC allows to shape both potential and kinetic energy of the system, as well as to inject damping and modified the interconnection structure of the system. This method relies on the solution of the socalled matching equation, which is set of partial differential equations (PDE). In general, solving the matching equation is not trivial and probably constitutes the most difficult step in the design. For the set-point regulation problem of fullyactuated mechanical system, can be solved by reshaping only the potential energy such that the close-loop potential energy has a minimum at the desired equilibrium state. If the control system needs to reject constant disturbance forces, it is necessary to add integral action. In the PH framework, the addition of integral action has been discussed in [10] and [11], and further specialised for fully-actuated mechanical systems in [12]. These methods for adding integral action do not apply to underactuated systems.

In this paper, we consider the control design problem for an underactuated robotic system. We first derive a model of the open-loop system in PHS form. We, then, design a set-point regulation controller with integral action using a state transformation and a dynamic extension. We proposed a desired closed loop system that retains the PHS form, and we add some degrees of freedom to solve the matching equation via dynamic augmentation. We show that the control system ensures regulation of the position references with internal stability. The addition of integral action ensures that the system is able to deal with problems such constant disturbance and provides robustness to modelling uncertainty [13].

\section{Mechanical Systems and Port Hamiltonian MODELS}

Some mechanical systems can be described using the Euler-Lagrange equation with dissipation:

$$
\frac{d}{d t}\left(\frac{\partial L}{\partial \dot{\mathbf{q}}}(\mathbf{q}, \dot{\mathbf{q}})\right)-\frac{\partial L}{\partial \mathbf{q}}(\mathbf{q}, \dot{\mathbf{q}})+\frac{\partial \mathcal{R}(\dot{\mathbf{q}})}{\partial \dot{\mathbf{q}}}=\boldsymbol{\tau},
$$

where $\mathbf{q}$ and $\dot{\mathbf{q}}$ are the $n$-dimensional vectors of generalised coordinates and velocities, and the $m$-dimensional vector $\tau$ is the vector of generalised forces. The function $\mathcal{R}(\dot{\mathbf{q}})$ is called the Rayleigh dissipation function, which satisfies

$$
\dot{\mathbf{q}}^{\top} \frac{\partial \mathcal{R}(\dot{\mathbf{q}})}{\partial \dot{\mathbf{q}}} \geq 0 .
$$


The Lagrangian $L(\mathbf{q}, \dot{\mathbf{q}})$ is the difference between the kinetic co-energy and the potential energy. For systems within the realm of classical mechanics, the Lagrangian takes the form

$$
L(\mathbf{q}, \dot{\mathbf{q}})=T(\mathbf{q}, \dot{\mathbf{q}})-V(\mathbf{q})=\frac{1}{2} \dot{\mathbf{q}}^{\top} \mathbf{M}(\mathbf{q}) \dot{\mathbf{q}}-V(\mathbf{q})
$$

where $\mathbf{M}(\mathbf{q})$ is the generalised mass matrix, which is symmetric and positive definite for all $\mathbf{q}$.

For these systems, the conjugate generalised momentum takes the form $\mathbf{p}=\frac{\partial L}{\partial \dot{\mathbf{q}}}=\mathbf{M}(\mathbf{q}) \dot{\mathbf{q}}$. Let us assume that the dissipation force takes the form $\boldsymbol{\tau}_{d}=\frac{\partial \mathcal{R}(\dot{\mathbf{q}})}{\partial \dot{\mathbf{q}}}=\mathbf{R}(\dot{\mathbf{q}}) \dot{\mathbf{q}}$, where $\mathbf{R}>0$. Using the momentum and the generalised coordinate vector, the set of $n$ second-order differential equations (1) can be transformed, using the Legendre's transformation, into a set of $2 n$ first-order differential equations [14]

$$
\begin{aligned}
& \dot{\mathbf{p}}=-\frac{\partial H(\mathbf{p}, \mathbf{q})}{\partial \mathbf{q}}-\mathbf{R} \frac{\partial H(\mathbf{p}, \mathbf{q})}{\partial \mathbf{p}}+\boldsymbol{\tau}, \\
& \dot{\mathbf{q}}=\frac{\partial H(\mathbf{p}, \mathbf{q})}{\partial \mathbf{p}},
\end{aligned}
$$

where the Hamiltonian is the sum of the kinetic energy and the potential energy: $H(\mathbf{p}, \mathbf{q})=T(\mathbf{q}, \dot{\mathbf{q}})+V(\mathbf{q})=$ $\frac{1}{2} \mathbf{p}^{\top} \mathbf{M}^{-1}(\mathbf{q}) \mathbf{p}+V(\mathbf{q})$. This function represents the total energy of the system. The equations (4) are called the Hamiltonian equations of motion.

In the control literature, the Hamiltonian model (4) has been generalised to what is known as a port-Hamiltonian system [15]:

$$
\begin{aligned}
\dot{\mathbf{x}} & =[\mathbf{J}(\mathbf{x})-\mathbf{R}(\mathbf{x})] \frac{\partial H(\mathbf{x})}{\partial \mathbf{x}}+\mathbf{g}(\mathbf{x}) \mathbf{u} \\
\mathbf{y} & =\mathbf{g}^{\top}(\mathbf{x}) \frac{\partial H(\mathbf{x})}{\partial \mathbf{x}}
\end{aligned}
$$

where $\mathbf{x} \in \mathbb{R}^{p}$ is the state vector and the Hamiltonian $H$ : $\mathbb{R}^{p} \rightarrow \mathbb{R}$ may represent the total energy stored in the system. The pair $\mathbf{u}, \mathbf{y} \in \mathbb{R}^{m}$ are the input and output variables. These are conjugate variables; that is, their inner product represents the power exchanged between the system and the environment. The function $\mathbf{J}(\mathbf{x})=-\mathbf{J}^{\top}(\mathbf{x})$ describes the power preserving interconnection structure through which the components of the system exchange energy. The symmetric function $\mathbf{R}(\mathbf{x}) \geq 0$ captures dissipative phenomena in the system. The function $\mathbf{g}(\mathbf{x})$ weighs the action of the input on the system and defines the conjugate output. From (5)-(6), it follows that

$$
\frac{d H}{d t}=\mathbf{y}^{\top} \mathbf{u}-\frac{\partial H^{\top}(\mathbf{x})}{\partial \mathbf{x}} \mathbf{R}(\mathbf{x}) \frac{\partial H(\mathbf{x})}{\partial \mathbf{x}} \leq \mathbf{y}^{\top} \mathbf{u},
$$

which shows passivity of the PHS model [6].

\section{Control of Mechanical System in Port HAMILTONIAN FRAMEWORK}

The system (4) can be written in the PHS form as follows

$$
\left[\begin{array}{c}
\dot{\mathbf{p}} \\
\dot{\mathbf{q}}
\end{array}\right]=\left\{\left[\begin{array}{cc}
\mathbf{0} & -\mathbf{I} \\
\mathbf{I} & \mathbf{0}
\end{array}\right]-\left[\begin{array}{cc}
\mathbf{D} & \mathbf{0} \\
\mathbf{0} & \mathbf{0}
\end{array}\right]\right\}\left[\begin{array}{c}
\frac{\partial H(\mathbf{p}, \mathbf{q})}{\partial \mathbf{p}} \\
\frac{\partial H(\mathbf{p}, \mathbf{q})}{\partial \mathbf{q}}
\end{array}\right]+\left[\begin{array}{c}
\mathbf{G} \\
\mathbf{0}
\end{array}\right] \boldsymbol{\tau},
$$

where

$$
H(\mathbf{p}, \mathbf{q})=T(\mathbf{p}, \mathbf{q})+V(\mathbf{q})=\frac{1}{2} \mathbf{p}^{\top} \mathbf{M}^{-1}(\mathbf{q}) \mathbf{p}+V(\mathbf{q}),
$$

$\mathbf{q} \in \mathbb{R}^{n}, \mathbf{p} \in \mathbb{R}^{n}$ and $\boldsymbol{\tau} \in \mathbb{R}^{m}$. The parameter $\mathbf{D}=\mathbf{D}^{\top} \in$ $\mathbb{R}^{n \times n}$ is the positive definite damping, and $\mathbf{G} \in \mathbb{R}^{n \times m}$ is the input coupling matrix. For typical underactuated systems $\operatorname{rank}(\mathbf{G})=m$ and $m<n$.

A controller for set point regulation can be designed such that in closed-loop the system retains its PHS form and the potential energy has a minimum at the desired equilibrium state. This ensures that the closed loop is passive, and thus inherits the robust properties of passive-based control system. Also, stability can be shown using the Hamiltonian as a Lyapunov candidate function.

The classical IDA-PBC technique aims to shape the energy of the system so that it has a minimum at the desired equilibrium. In addition, the new interconnection matrix and dissipation function can be assigned for the closed loop [8]. In particular, the desired closed loop for underactuated mechanical systems takes the form (see e.g. [16])

$$
\left[\begin{array}{c}
\dot{\mathbf{p}} \\
\dot{\mathbf{q}}
\end{array}\right]=\left[\begin{array}{cc}
-\mathbf{D}_{\mathbf{d}} & -\mathbf{M}_{\mathbf{d}}(\mathbf{q}) \mathbf{M}^{-1}(\mathbf{q}) \\
\mathbf{M}^{-1}(\mathbf{q}) \mathbf{M}_{\mathbf{d}}(\mathbf{q}) & \mathbf{0}
\end{array}\right]\left[\begin{array}{l}
\frac{\partial H_{d}}{\partial \mathbf{p}} \\
\frac{\partial H_{d}}{\partial \mathbf{q}}
\end{array}\right]
$$

where $\mathbf{D}_{\mathbf{d}}=\mathbf{D}_{\mathbf{d}}{ }^{\top}>0$ is the desired damping, and the closed-loop Hamiltonian is

$$
H_{d}(\mathbf{p}, \mathbf{q})=\frac{1}{2} \mathbf{p}^{\top} \mathbf{M}_{\mathbf{d}}^{-1}(\mathbf{q}) \mathbf{p}+V_{d}(\mathbf{q}),
$$

and $\mathbf{M}_{\mathbf{d}}(\mathbf{q})=\mathbf{M}_{\mathbf{d}}{ }^{\top}(\mathbf{q})>0$ is the desired mass matrix. The potential energy $V_{d}(\mathbf{q})$ is chosen to have a minimum at the desired equilibrium.

The problem is to find a control $\tau$ that renders the open loop (8) into the desired PHS (10). The classical procedure to solve this problem is by matching the state equations from (8) and (10). This result in the following so-called matching equation

$$
\begin{aligned}
\mathbf{G}^{\perp}\left\{\mathbf{D M}^{-1}(\mathbf{q}) \mathbf{p}-\mathbf{D}_{\mathbf{d}} \mathbf{M}_{d}^{-1}(\mathbf{q}) \mathbf{p}\right. & \\
& \left.+\frac{\partial H}{\partial \mathbf{q}}-\mathbf{M}_{\mathbf{d}}(\mathbf{q}) \mathbf{M}^{-1}(\mathbf{q}) \frac{\partial H_{d}}{\partial \mathbf{q}}\right\}=\mathbf{0},
\end{aligned}
$$

where $\mathbf{G}^{\perp}$ is any full-rank left annihilator of $\mathbf{G}$, that is, $\mathbf{G}^{\perp} \mathbf{G}=\mathbf{0}$ and $\operatorname{rank}\left(\mathbf{G}^{\perp}\right)=n-m$. If (12) is satisfied, the control law is readily computed as follows

$$
\begin{aligned}
\boldsymbol{\tau}=\left(\mathbf{G}^{\top} \mathbf{G}\right)^{-1} \mathbf{G}^{\top} & \left\{\mathbf{D M}^{-1}(\mathbf{q}) \mathbf{p}-\mathbf{D}_{\mathbf{d}} \mathbf{M}_{\mathbf{d}}^{-1}(\mathbf{q}) \mathbf{p}\right. \\
+ & \left.\frac{\partial H}{\partial \mathbf{q}}-\mathbf{M}_{\mathbf{d}}(\mathbf{q}) \mathbf{M}^{-1}(\mathbf{q}) \frac{\partial H_{d}}{\partial \mathbf{q}}\right\} .
\end{aligned}
$$

For underactuated system, (12) consists of $n-m$ partial differential equations on $H_{d}$. This set of PDE admits solutions for a family of achievable desired Hamiltonian functions, which in general is difficult to find. From this family, we choose a particular solution that satisfied the 
minimum requirement, that is, $\left(\mathbf{0}, \mathbf{q}^{*}\right)=\arg \min H_{d}$, where $\mathbf{q}^{*}$ is the desired equilibrium position. The research in the literature aims to provide techniques for solving the matching equation for underactuated systems (see e.g. [17], [16], [18]).

It is known that passivity control designs are robust to parametric uncertainties and unmodelled dynamics, in the sense that stability is preserved provided that the closedloop is passive. However, external disturbances can shift the desired equilibrium or, even worse, cause the system to be unstable. A standard solution to deal with external disturbances is the addition of integral action in the loop [13]. For PH control systems, integral control can be designed using the approaches in [19], [10], [11], which have been specialised for mechanical systems in [12]. However, the these methods do not apply to underactuated systems in general. Hence, in the next section, we consider this particular problem.

\section{Set Point Regulation with Integral Action OF AN UNDERACTUATED ROBOTIC SYSTEM}

\section{A. PHS Modelling}

We consider the robot manipulator mounted on a flexible base shown in Figure 1 [20]. The base only moves in the vertical direction, and its position is described by $q_{1}$, which represent the displacement of the base from its natural rest position when the tool is unmounted. The robot arm has two degrees of freedom, which correspond to the rotation $q_{2}$ and extension $q_{3}$ of the arm. The control torque $\tau_{m}$ drives the arm, and the control force $F_{A}$ acts to extend the arm. The task of the robot is to move a heavy tool of mass $m_{T}$ to a particular position in the workspace. The port-Hamiltonian model of the system has the form (8), with mass matrix

$\mathbf{M}(\mathbf{q})=\left[\begin{array}{ccc}m_{B}+m_{T} & m_{T}\left(q_{3}+\ell\right) \cos q_{2} & m_{T} \sin q_{2} \\ m_{T}\left(q_{3}+\ell\right) \cos q_{2} & m_{T}\left(q_{3}+\ell\right)^{2} & 0 \\ m_{T} \sin q_{2} & 0 & m_{T}\end{array}\right]$,

and dissipation matrix $\mathbf{D}=\operatorname{diag}\left(b_{B}, b_{M}, b_{A}\right)$. The Hamiltonian function is

$$
H(\mathbf{p}, \mathbf{q})=\frac{1}{2} \mathbf{p}^{\top} \mathbf{M}^{-1}(\mathbf{q}) \mathbf{p}+V(\mathbf{q})
$$

with potential energy $V(\mathbf{q})=\frac{1}{2} k_{A} q_{3}^{2}+\frac{1}{2} k_{B} q_{1}^{2}+m_{T} g q_{1}+$ $m_{T} g\left(q_{3}+\ell\right) \sin q_{2}$. The input coupling matrix $\mathbf{G}$ and its left-annihilator $\mathbf{G}^{\perp}$ are given by

$$
\mathbf{G}=\left[\begin{array}{ll}
0 & 0 \\
1 & 0 \\
0 & 1
\end{array}\right], \quad \mathbf{G}^{\perp}=\left[\begin{array}{lll}
1 & 0 & 0
\end{array}\right] .
$$

\section{B. Control Design}

The control task is to regulate the position $q_{2}$ and $q_{3}$ to constant references $q_{2}^{*}$ and $q_{3}^{*}$. The control system should be designed to compensate for motion of the base, while positioning the end effector at the desired references, and rejecting external constant disturbance.

We proposed a control design that incorporates both set point regulation and integral action for the underactuated

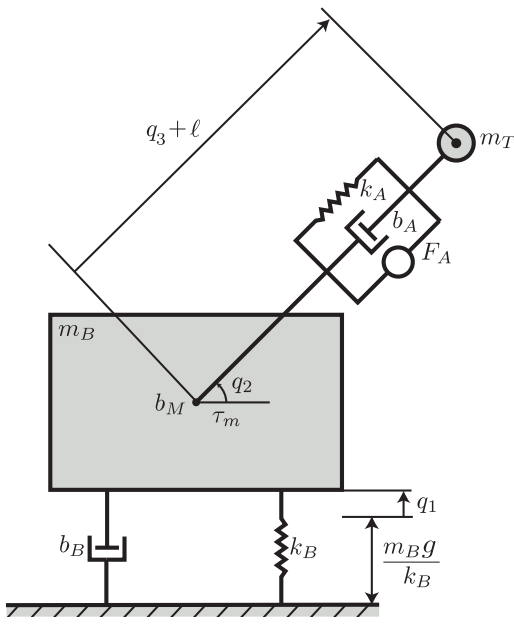

Fig. 1. Robotic manipulator mounted on a flexible base.

robotic system shown in Figure 1. Instrumental for our design are the following tools:

i) (Coordinates transformation) A change of coordinates

$$
\begin{aligned}
& \mathbf{z}_{p}=\mathbf{p}-\mathbf{p}^{*}\left(\mathbf{p}, \mathbf{q}, q_{1}^{*}, q_{2}^{*}, q_{3}^{*}\right) \\
& \mathbf{z}_{q}=\mathbf{q}-\mathbf{q}^{*}\left(q_{1}^{*}, q_{2}^{*}, q_{3}^{*}\right)
\end{aligned}
$$

where $\mathbf{p}^{*}$ and $\mathbf{q}^{*}$ are functions of the states and references signals. These functions will be chosen to ensure the stability of the closed loop. The form of the change of coordinates is inspired in the state transformation proposed in [21].

ii) (Closed-loop PHS) We then proposed a desired closedloop PHS system in the new coordinates $\mathbf{z}$, which include additional states $\mathbf{z}_{e} \in \mathbb{R}^{2}$ for integral action. The closed loop preserves the classical form (10) but with full dissipation, which has the form

$$
\begin{gathered}
{\left[\begin{array}{c}
\dot{\mathbf{z}}_{p} \\
\dot{\mathbf{z}}_{q} \\
\dot{\mathbf{z}}_{e}
\end{array}\right]=\left\{\left[\begin{array}{ccc}
0 & -\mathbf{M}_{\mathbf{d}} \mathbf{M}^{-1}(\mathbf{q}) & -\mathbf{Q}_{1}^{\top} \\
\mathbf{M}^{-1}(\mathbf{q}) \mathbf{M}_{\mathbf{d}} & \mathbf{0} & -\mathbf{K}_{\mathbf{I}} \\
\mathbf{Q}_{1} & \mathbf{K}_{\mathbf{I}}^{\top} & 0
\end{array}\right]-\right.} \\
\left.\left[\begin{array}{ccc}
\mathbf{B}_{\mathbf{p z}} & 0 & 0 \\
0 & \mathbf{B}_{\mathbf{q z}} & 0 \\
0 & 0 & \mathbf{B}_{\mathbf{z e}}
\end{array}\right]\right\}\left[\begin{array}{l}
\frac{\partial H_{d z}\left(\mathbf{z}_{p}, \mathbf{z}_{q}, \mathbf{z}_{e}\right)}{\partial \mathbf{z}_{p}} \\
\frac{\partial H_{d z}\left(\mathbf{z}_{p}, \mathbf{z}_{q}, \mathbf{z}_{e}\right)}{\partial \mathbf{z}_{q}} \\
\frac{\partial H_{d z}\left(\mathbf{z}_{p}, \mathbf{z}_{q}, \mathbf{z}_{e}\right)}{\partial \mathbf{z}_{e}}
\end{array}\right]
\end{gathered}
$$

where $\mathbf{B}_{\mathbf{p z}} \in \mathbb{R}^{3 \times 3}, \mathbf{B}_{\mathbf{q z}} \in \mathbb{R}^{3 \times 3}$, and $\mathbf{B}_{\mathbf{z e}} \in \mathbb{R}^{2 \times 2}$ are positive definite and symmetric matrices. They represent the damping in the coordinates $\mathbf{z}_{p}, \mathbf{z}_{q}$, and $\mathbf{z}_{e}$ respectively. The matrices $\mathbf{Q}_{1} \in \mathbb{R}^{2 \times 3}$ and $\mathbf{K}_{\mathbf{I}} \in \mathbb{R}^{3 \times 2}$ set the integral action, as we will show later. The desired closed-loop Hamiltonian in $\mathbf{z}$ coordinates is

$$
H_{d z}=\frac{1}{2} \mathbf{z}_{p}^{\top} \mathbf{M}_{\mathbf{d}}^{-\mathbf{1}} \mathbf{z}_{p}+\frac{1}{2} \mathbf{z}_{q}^{\top} \mathbf{K}_{\mathbf{q}} \mathbf{z}_{q}+\frac{1}{2} \mathbf{z}_{e}^{\top} \mathbf{K}_{\mathbf{e}} \mathbf{z}_{e}
$$

where $\mathbf{M}_{\mathbf{d}}$ is the desired closed-loop mass matrix, which is a constant, positive definite and symmetric matrix. The constant and positive definite matrices $\mathbf{K}_{\mathbf{q}} \in \mathbb{R}^{3 \times 3}$ and $\mathbf{K}_{\mathbf{e}} \in \mathbb{R}^{2 \times 2}$, are design parameters. 
iii) (Dynamic extension) We also add states $q_{1}^{*}$ and $\dot{q}_{1}^{*}$ in the controller, whose dynamics can be described as

$$
\mathbf{A} \ddot{q}_{1}^{*}+\mathbf{B} \dot{q}_{1}^{*}+\mathbf{C} q_{1}^{*}=\mathbf{F}\left(\mathbf{z}, q_{2}^{*}, q_{3}^{*}\right)
$$

where $\mathbf{A}, \mathbf{B}$ and $\mathbf{C}$ are positive constants. The function $\mathbf{F}$ is bounded when its arguments are bounded, and $\mathbf{F}\left(\mathbf{0}, q_{2}^{*}, q_{3}^{*}\right)=c$, where $c$ is a constant.

The design consists in finding a control law $\tau$ such that the closed loop in coordinates $\mathbf{z}=\left[\mathbf{z}_{p}, \mathbf{z}_{q}, \mathbf{z}_{e}\right]$ has the form (18), and the dynamics of $q_{1}^{*}$ and $\dot{q}_{1}^{*}$ satisfies (20). Under this conditions, it can be proved that since (18) is a PHS with a energy minimum at the origin, then the trajectories $\mathbf{z}(t)$ converge to zero, that is, $\mathbf{z} \rightarrow 0$, hence $\mathbf{F}\left(\mathbf{z}, q_{2}^{*}, q_{3}^{*}\right) \rightarrow c$. Finally, the stability of the full control system is ensured by showing bounded-input-bounded-state (BIBS) and convergent-inputconvergent-state (CICS) properties of the system (20), with input $\mathbf{F}$ and states $q_{1}^{*}$ and $\dot{q}_{1}^{*}$. Indeed, since $\mathbf{z} \rightarrow 0$ and $q_{1}^{*}$ converge to a constant value, then $\mathbf{q} \rightarrow \mathbf{q}^{*}=\left[q_{1}^{*}, q_{2}^{*}, q_{3}^{*}\right]$ and $\mathbf{p} \rightarrow \mathbf{0}$, as desired.

To design the controller, we first make the time derivative of the change of coordinate (17), and then we replace the derivative of the states by their corresponding state equations from (8) and (18), as follows

$$
\begin{aligned}
\dot{\mathbf{q}}-\dot{\mathbf{q}}^{*} & =\mathbf{M}(\mathbf{q})^{-1} \mathbf{p}-\dot{\mathbf{q}}^{*} \\
& \equiv \dot{\mathbf{z}}_{q} \\
& =\mathbf{M}(\mathbf{q})^{-1} \mathbf{z}_{\mathbf{p}}-\mathbf{K}_{\mathbf{I}} \mathbf{K}_{\mathbf{e}} \mathbf{z}_{e}-\mathbf{B}_{\mathbf{q z}} \mathbf{K}_{\mathbf{q}} \mathbf{z}_{q} \\
& =\mathbf{M}(\mathbf{q})^{-1}\left(\mathbf{p}-\mathbf{p}^{*}\right)-\mathbf{K}_{\mathbf{I}} \mathbf{K}_{\mathbf{e}} \mathbf{z}_{e}-\mathbf{B}_{\mathbf{q z}} \mathbf{K}_{\mathbf{q}} \mathbf{z}_{q}
\end{aligned}
$$

which is satisfied by

$$
\mathbf{p}^{*}=\mathbf{M}(\mathbf{q}) \dot{\mathbf{q}}^{*}-\mathbf{M}(\mathbf{q}) \mathbf{K}_{\mathbf{I}} \mathbf{K}_{\mathbf{e}} \mathbf{z}_{e}-\mathbf{M}(\mathbf{q}) \mathbf{B}_{\mathbf{q z}} \mathbf{K}_{\mathbf{q}} \mathbf{z}_{q},
$$

then the dynamic of the position error $\left(\mathbf{q}-\mathbf{q}^{*}\right)$ can be represented by the desired state equation of $\mathbf{z}_{q}$ in (18).

Second, we make the time derivative of the change of coordinates (16), and then we replace the derivatives of the states by their corresponding state equations from (8) and (18), as follows

$$
\begin{aligned}
\dot{\mathbf{p}}-\dot{\mathbf{p}}^{*} & =-\mathbf{D} \frac{\partial H}{\partial \mathbf{p}}-\frac{\partial H}{\partial \mathbf{q}}+\mathbf{G} \tau-\dot{\mathbf{p}}^{*} \\
& \equiv \dot{\mathbf{z}}_{p} \\
& =-\mathbf{M}_{d} \mathbf{M}^{-1}(\mathbf{q}) \frac{\partial H_{d z}}{\partial \mathbf{z}_{q}}-\mathbf{Q}_{1}^{\top} \frac{\partial H_{d z}}{\mathbf{z}_{e}}-\mathbf{B}_{p z} \frac{\partial H_{d z}}{\partial \mathbf{z}_{p}}
\end{aligned}
$$

Multiplying (23) by $\mathbf{G}^{\perp}$ yields the matching equation

$$
\begin{array}{r}
\mathbf{G}^{\perp}\left\{\mathbf{D} \frac{\partial H}{\partial \mathbf{p}}+\frac{\partial H}{\partial \mathbf{q}}-\mathbf{B}_{p z} \frac{\partial H_{d z}}{\partial \mathbf{z}_{p}}-\mathbf{M}_{\mathbf{d}} \mathbf{M}(\mathbf{q})^{-1} \frac{\partial H_{d z}}{\partial \mathbf{z}_{q}}\right. \\
\left.-\mathbf{Q}_{1}^{\top} \frac{\partial H_{d z}}{\mathbf{z}_{e}}+\dot{\mathbf{p}}^{*}\right\}=\mathbf{0}
\end{array}
$$

The function $q_{1}^{*}$, and its time derivatives $\dot{q}_{1}^{*}$ and $\ddot{q}_{1}^{*}$, which have not yet been selected, may help to solve the matching equation (24). Indeed, we can replace $\dot{\mathbf{p}}^{*}$ by the time derivative of (22) in (24), and then solve it for $q_{1}^{*}$. The matching equation (24) is solved if $q_{1}^{*}$ satisfies

$$
\mathbf{A} \ddot{q}_{1}^{*}+\mathbf{B} \dot{q}_{1}^{*}+\mathbf{C} q_{1}^{*}=\mathbf{F}\left(\mathbf{z}, q_{2}^{*}, q_{3}^{*}\right),
$$

where $\mathbf{A}=m_{B}+m_{T}, \mathbf{B}=b_{B}$ and $\mathbf{C}=k_{B}$ are positive constants. The function $\mathbf{F}$ is continuous and bounded for all constants $q_{2}^{*}$ and $q_{3}^{*}$, and all bounded $\mathbf{z}$ in the robot workspace, which implies $q_{3}+\ell>0$. In addition, $\mathbf{F}$ converges to $\mathbf{F}\left(\mathbf{0}, q_{2}^{*}, q_{3}^{*}\right)=-m_{T} g$ when $\mathbf{z} \rightarrow 0$.

We add the dynamics (25) in the controller to compute the signals $\ddot{q}_{1}^{*}, \dot{q}_{1}^{*}$, and $q_{1}^{*}$, which are needed in the control law. Note that $q_{1}^{*}$ and $\dot{q}_{1}^{*}$ are not external references, but states of the controller.

The control law is computed from (23) as follows

$$
\begin{array}{r}
\boldsymbol{\tau}=\left(\mathbf{G}^{\top} \mathbf{G}\right)^{-1} \mathbf{G}^{\top}\left\{\mathbf{D} \frac{\partial H}{\partial \mathbf{p}}+\frac{\partial H}{\partial \mathbf{q}}-\mathbf{B}_{\mathbf{p z}} \frac{\partial H_{d z}}{\partial \mathbf{z}_{p}}-\right. \\
\left.\mathbf{M}_{\mathbf{d}} \mathbf{M}(\mathbf{q})^{-1} \frac{\partial H_{d z}}{\partial \mathbf{z}_{q}}-\mathbf{Q}_{1}^{\top} \frac{\partial H_{d z}}{\mathbf{z}_{e}}+\dot{\mathbf{p}}^{*}\right\} .
\end{array}
$$

where $\dot{\mathbf{p}}^{*}$ is replaced by the time derivative of (22), and the time derivative of the states by their corresponding state equations.

The integral action on the system is obtained through states $\mathbf{z}_{e}$. The dynamics of the integral action is

$$
\begin{aligned}
\dot{\mathbf{z}}_{e}= & \mathbf{Q}_{1} \frac{\partial H_{d z}}{\partial \mathbf{z}_{p}}+\mathbf{K}_{\mathbf{I}}^{\top} \frac{\partial H_{d z}}{\partial \mathbf{z}_{q}}-\mathbf{B}_{\mathbf{z e}} \frac{\partial H_{d z}}{\partial \mathbf{z}_{e}} \\
= & \mathbf{Q}_{1} \mathbf{M}_{\mathbf{d}}{ }^{-1} \mathbf{z}_{p}+\mathbf{K}_{\mathbf{I}} \mathbf{K}_{\mathbf{q}} \mathbf{z}_{q}-\mathbf{B}_{\mathbf{z e}} \mathbf{K}_{\mathbf{e}} \mathbf{z}_{e} \\
= & \mathbf{Q}_{1} \mathbf{M}_{\mathbf{d}}{ }^{-1}\left[\mathbf{p}-\mathbf{p}^{*}\right]+\mathbf{K}_{\mathbf{I}} \mathbf{K}_{\mathbf{q}} \mathbf{z}_{q}-\mathbf{B}_{\mathbf{z e}} \mathbf{K}_{\mathbf{e}} \mathbf{z}_{e} \\
= & \mathbf{Q}_{1} \mathbf{M}_{\mathbf{d}}{ }^{-1}\left[\mathbf{p}-\mathbf{M}(\mathbf{q}) \dot{\mathbf{q}}^{*}+\mathbf{M}(\mathbf{q}) \mathbf{B}_{\mathbf{p} \mathbf{z}} \mathbf{K}_{\mathbf{q}} \mathbf{z}_{q}\right]+ \\
& +\mathbf{K}_{\mathbf{I}} \mathbf{K}_{\mathbf{q}} \mathbf{z}_{q}
\end{aligned}
$$

where $\mathbf{Q}_{1}=\mathbf{B}_{\mathbf{z e}}\left(\mathbf{K}_{\mathbf{I}}^{\top} \mathbf{K}_{\mathbf{I}}\right)^{-1} \mathbf{K}_{\mathbf{I}}^{\top} \mathbf{M}(\mathbf{q})^{-1} \mathbf{M}_{\mathbf{d}}$ is chosen to eliminate $\mathbf{z}_{e}$ from the integral action dynamics. Note that at steady state, $\mathbf{z}_{q}$ has to be zero since $\mathbf{p}$ and $\dot{\mathbf{q}}^{*}$ are zero. Thus, integral action ensures set-point regulation of $\mathbf{q}$.

\section{Stability Analysis}

The procedure in the previous section allows us to write the closed-loop dynamics as the PHS (18) plus the dynamics (25). In this section, we study the stability of this closed loop.

Proposition 1: The PHS (18) has an asymptotic stable equilibrium at $\mathbf{z}=\mathbf{0}$. Thus, momenta $\mathbf{p}$ and positions $\mathbf{q}$, converge to their references $\mathbf{p}^{*}$ and $\mathbf{q}^{*}$ respectively.

Proof: The Hamiltonian $H_{d z}$ (19) has a minimum at $\mathbf{z}=0$, which is also an equilibrium point since $\left.\frac{\partial H_{d z}}{\partial \mathbf{z}}\right|_{\mathbf{z}=\mathbf{0}}=$ $\mathbf{0}$. We use $H_{d z}$ as a Lyapunov candidate function, and we compute its time derivative along the solution of (18) as follows

$$
\begin{aligned}
\dot{H}_{d z} & =\frac{\partial^{\top} H_{d z}}{\partial \mathbf{z}_{p}} \dot{\mathbf{z}}_{p}+\frac{\partial^{\top} H_{d z}}{\partial \mathbf{z}_{q}} \dot{\mathbf{z}}_{q}+\frac{\partial^{\top} H_{d z}}{\partial \mathbf{z}_{e}} \dot{\mathbf{z}}_{e} \\
& =-\frac{\partial^{\top} H_{d z}}{\partial \mathbf{z}_{p}} \mathbf{B}_{\mathbf{p z}} \frac{\partial H_{d z}}{\partial \mathbf{z}_{p}}-\frac{\partial^{\top} H_{d z}}{\partial \mathbf{z}_{q}} \mathbf{B}_{\mathbf{q z}} \frac{\partial H_{d z}}{\partial \mathbf{z}_{q}} \\
& -\frac{\partial^{\top} H_{d z}}{\partial \mathbf{z}_{e}} \mathbf{B}_{\mathbf{z e}} \frac{\partial H_{d z}}{\partial \mathbf{z}_{e}}=-\mathbf{z}_{p}^{\top} \mathbf{M}_{\mathbf{d}}^{-\mathbf{1}} \mathbf{B}_{\mathbf{p z}} \mathbf{M}_{\mathbf{d}}^{-\mathbf{1}} \mathbf{z}_{p}- \\
& -\mathbf{z}_{q}^{\top} \mathbf{K}_{\mathbf{q}} \mathbf{B}_{\mathbf{q z}} \mathbf{K}_{\mathbf{q}} \mathbf{z}_{q}-\mathbf{z}_{e}^{\top} \mathbf{K}_{\mathbf{e}} \mathbf{B}_{\mathbf{z e}} \mathbf{K}_{\mathbf{e}} \mathbf{z}_{e}<0,
\end{aligned}
$$


which establishes the asymptotic stability of the the equilibrium $\mathbf{z}=\mathbf{0}$. Since the trajectories $\mathbf{z}(t) \rightarrow \mathbf{0}$ as $t \rightarrow \infty$, then trajectories $\mathbf{p}(t) \rightarrow \mathbf{p}^{*}$ and $\mathbf{q}(t) \rightarrow \mathbf{q}^{*}$, as desired.

Proposition 2: Consider the dynamics (25) with bounded input $\mathbf{F}\left(\mathbf{z}, q_{2}^{*}, q_{3}^{*}\right)$. Assume that $\mathbf{F}\left(\mathbf{z}, q_{2}^{*}, q_{3}^{*}\right) \rightarrow$ $\mathbf{F}\left(\mathbf{0}, q_{2}^{*}, q_{3}^{*}\right)=-m_{T} g$ as $\mathbf{z} \rightarrow \mathbf{0}$. Then, the trajectories of the states $\dot{q}_{1}^{*}(t)$ and $q_{1}^{*}(t)$ converge to 0 and $\frac{-m_{T} g}{k_{B}}$.

Proof: Using the state transformation $v_{1}=\mathbf{A} \dot{q}_{1}^{*}$ and $v_{2}=q_{1}^{*}-\frac{\mathbf{F}\left(\mathbf{0}, q_{2}^{*}, q_{3}^{*}\right)}{\mathbf{C}}$, the dynamics (25) can be written as

$$
\left[\begin{array}{c}
\dot{v_{1}} \\
\dot{v_{2}}
\end{array}\right]=\left[\begin{array}{cc}
-\mathbf{B} & 1 \\
1 & 0
\end{array}\right]\left[\begin{array}{c}
\frac{\partial W\left(v_{1}, v_{2}\right)}{\partial v_{1}} \\
\frac{\partial W\left(v_{1}, v_{2}\right)}{\partial v_{2}}
\end{array}\right]+\left[\begin{array}{c}
\tilde{\mathbf{F}}\left(\mathbf{z}, q_{2}^{*}, q_{3}^{*}\right) \\
0
\end{array}\right]
$$

with $W\left(v_{1}, v_{2}\right)=\frac{1}{2} \mathbf{A}^{-1} v_{1}^{2}+\frac{1}{2} \mathbf{C} v_{2}^{2}$, and $\tilde{\mathbf{F}}\left(\mathbf{z}, q_{2}^{*}, q_{3}^{*}\right)=$ $\mathbf{F}\left(\mathbf{z}, q_{2}^{*}, q_{3}^{*}\right)-\mathbf{F}\left(\mathbf{0}, q_{2}^{*}, q_{3}^{*}\right)$, which is bounded and $\tilde{\mathbf{F}}\left(\mathbf{0}, q_{2}^{*}, q_{3}^{*}\right)=0$. For the unforced case, i.e. $\tilde{\mathbf{F}}=0$, then, the equilibrium point $\left(v_{1}, v_{2}\right)=(0,0)$ is asymptotically stable. Indeed, using $W$ as a Lyapunov candidate function, it yields

$$
\dot{W}=-\mathbf{B} v_{1}^{2} \leq 0,
$$

and since the maximum invariance, under the dynamics (29), contained in $\mathcal{S}=\left\{\left(v_{1}, v_{2}\right) \mid \dot{W}\left(v_{1}, v_{2}\right)=0\right\}$ is the origin, then $\left(v_{1}, v_{2}\right)=(0,0)$ is an asymptotically stable equilibrium. The stability property is global since $W$ is radially unbounded. Note that the system (29) is linear and asymptotically stable, then it is input-to-state-stable (ISS) (see e.g. [13, page 174]). ISS property ensures that the states are bounded when the inputs are bounded, and that the states converge to zero when the input converges to zero. Then, it follows that $\tilde{\mathbf{F}}\left(\mathbf{z}, q_{2}^{*}, q_{3}^{*}\right) \rightarrow 0$ as $\mathbf{z} \rightarrow \mathbf{0} \Rightarrow\left(v_{1}, v_{2}\right) \rightarrow(0,0)$, and that the trajectories $v_{1}(t)$ and $v_{2}(t)$ are bounded since $\tilde{\mathbf{F}}$ is bounded. Thus, the trajectories $\dot{q}_{1}^{*}(t)$ and $q_{1}^{*}(t)$ are bounded and converge to 0 and $\frac{-m_{T} g}{k_{B}}$ respectively.

Corollary 1: Consider the robotic system shown in Figure 1 with Hamiltonian (14), and control law (26) with integral action (27) and extended dynamics (25). Then, the closed-loop system ensures output regulation with internal stability.

Proof: The proof follows from the cascade representation of the closed loop. Indeed, the dynamics of the control system can be written as the cascade of the PHS (18) with the extended dynamics (25). Proposition 1 shows that the states $\mathbf{z}$ converge to zero, and then $(\mathbf{p}, \mathbf{q}) \rightarrow\left(\mathbf{p}^{*}, \mathbf{q}^{*}\right)$. Proposition 2 shows that $\dot{q}_{1}^{*}(t)$ and $q_{1}^{*}(t)$ are bounded and converge to 0 and $\frac{-m_{T} g}{k_{B}}$ as $\mathbf{z} \rightarrow \mathbf{0}$. Then, from (22) and since $\dot{\mathbf{q}}^{*} \rightarrow \mathbf{0}$ it follows that $\mathbf{p}^{*} \rightarrow \mathbf{0}$. The vector $\mathbf{q}^{*}$ converges to $\left(\frac{-m_{T} g}{k_{B}}, q_{2}^{*}, q_{3}^{*}\right)$, where $q_{2}^{*}$ and $q_{3}^{*}$ are the desired angular position and extension of the robot arm.

\section{Simulation Results}

In this section, we present simulations to evaluate the performance of the control system. The robot manipulator and controller parameters are given in the Appendix. The initial conditions of the system are $\mathbf{p}(0)=\mathbf{q}(0)=\mathbf{z}_{e}(0)=$ 0 , and $q_{1}^{*}(0)=\dot{q}_{1}^{*}(0)=0$. The target position is located at $x=4 \mathrm{~m}$ and $y=3 \mathrm{~m}$ that corresponds to $q_{2}^{*}=0.7 \mathrm{rad}$ and $q_{3}^{*}=4.2 \mathrm{~m}$. We also inject a constant torque disturbance of $500 \mathrm{~N} \cdot \mathrm{m}$ in the arm at $t=20 \mathrm{~s}$, and a constant force disturbance of $500 \mathrm{~N}$ into coordinate $q_{3}$ at $t=40 \mathrm{~s}$.

Figure 2 shows the time history of the configuration variables $\mathbf{q}$ and the desired position $\mathbf{q}^{*}$. As we can see, the positions converge to their desired references, as predicted by the theory. It can also be appreciated that the control action drives the position $q_{2}$ and $q_{3}$ to their reference despite the action of constant disturbances. The controller states that produce the integral action are shown in right column of Figure 3. The left column of Figure 3 shows the position errors of the tool in the workspace $x y$. The tool is positioned at the desired point in the workspace as the error converges quickly to zero. Moreover, the control system rejects constant disturbances added in the coordinates $q_{2}$ and $q_{3}$.

The plots show that both the control torque and control force are smooth and between acceptable bounds (see Figure 4). Furthermore, the controller produces the control inputs needed to reject the disturbance at 20 s and 40 s while keeping set-point regulation.
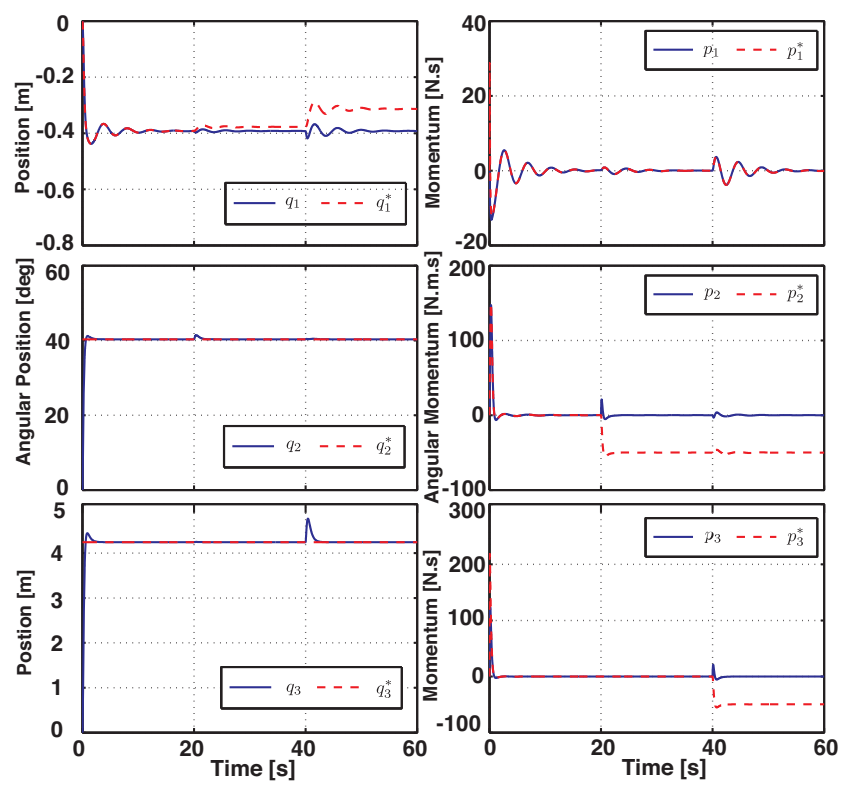

Fig. 2. Configuration variables $\mathbf{q}$, desired position $\mathbf{q}^{*}$, conjugate momenta $\mathbf{p}$ and desired momenta $\mathbf{p}^{*}$.

\section{CONCLUSION}

In this paper, we propose a PHS design for set-point position regulation and integral control for an underactuated robotic system. This design extends the methods available for PH mechanical system by allowing add integral action on non-passive outputs for an underactuated mechanical systems. Also, the ability of injecting damping in all the coordinates of the closed-loop PHS is a key characteristic that makes the Hamiltonian a strict Lyapunov function in the transformed coordinates $\mathbf{z}$. This can be useful to study the robust properties of the closed loop in term of ISS theory, and design tracking controllers for time-varying references. These topics are the subject of our current research. 

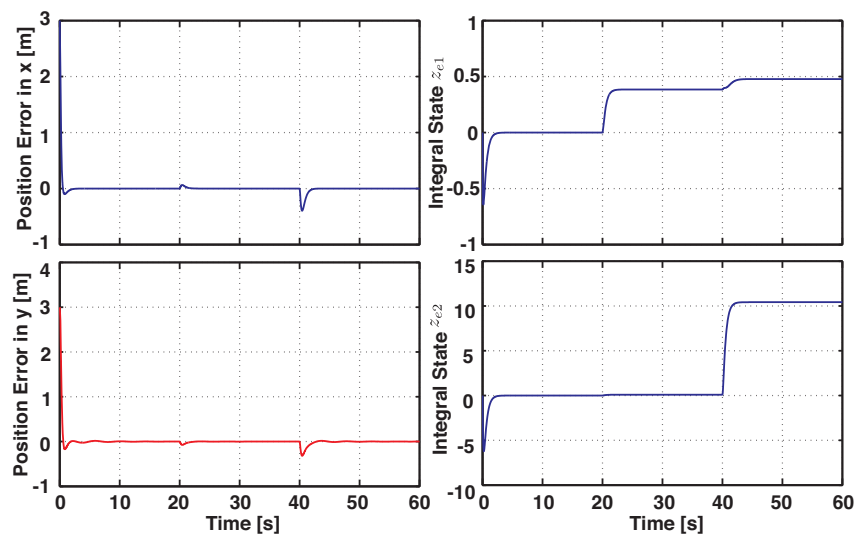

Fig. 3. Tool position errors in xy-workspace (left), and controller states for integral action (right).

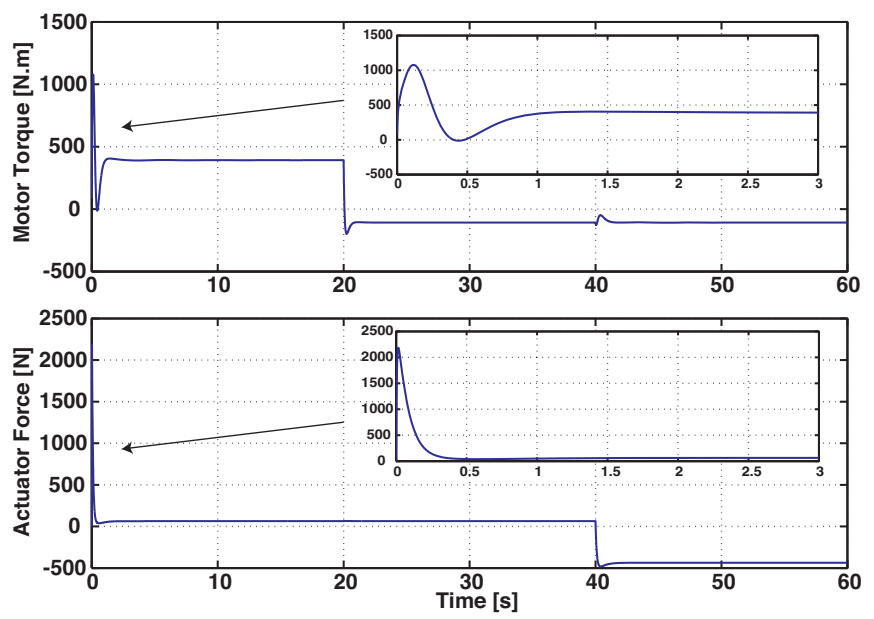

Fig. 4. Torque and force control commands.

\section{APPENDIX}

The plant parameters are given by $m_{B}=100 \mathrm{~kg}, m_{T}=$ $10 \mathrm{~kg}, \ell=1 \mathrm{~m}, k_{A}=0 \mathrm{~N} / \mathrm{m}, k_{B}=150 \mathrm{~N} / \mathrm{m}, b_{B}=50$ $\mathrm{Ns} / \mathrm{m}, b_{M}=1 \mathrm{Nms} / \mathrm{rad}$ and $b_{A}=50 \mathrm{Ns} / \mathrm{m}$. The values of the controller parameters are $\mathbf{M}_{\mathbf{d}}=\operatorname{diag}(a 1, a 4, a 6)$, $\mathbf{B}_{\mathbf{p z}}=\operatorname{diag}\left(b_{p 1}, b_{p 4}, b_{p 6}\right), \mathbf{B}_{\mathbf{q z}}=\operatorname{diag}\left(b_{q 1}, b_{q 4}, b_{q 6}\right)$, $\mathbf{B}_{\mathbf{z e}}=\operatorname{diag}\left(b_{z 1}, b_{z 2}\right), \mathbf{K}_{\mathbf{q}}=\operatorname{diag}\left(k_{q 1}, k_{q 2}, k_{q 3}\right), \mathbf{K}_{\mathbf{e}}=$ $\operatorname{diag}\left(k_{e 1}, k_{e 2}\right)$ and $\mathbf{K}_{\mathbf{I}}$ is given as

$$
\mathbf{K}_{\mathbf{I}}=\left[\begin{array}{cc}
0 & 0 \\
k_{i 1} & 0 \\
0 & k_{i 2}
\end{array}\right]
$$

\section{REFERENCES}

[1] F. Bullo and A. Lewis, Geometric control of mechanical systems. New York-Heidelberg-Berlin: Springer Verlag, 2004, vol. 49.

[2] I. Hussein and A. Bloch, "Optimal control of underactuated nonholonomic mechanical systems," IEEE Transactions on Automatic Control, vol. 53, pp. 668-682, 2008.

[3] P. X. M. La Hera, A. S. Shiriaev, L. B. Freidovich, U. Mettin, and S. V. Gusev, "Stable walking gaits for a three-link planar biped robot with one actuator," IEEE Transactions on Robotics, pp. 1-13, 2013.
TABLE I

CONTROLLER PARAMETERS.

\begin{tabular}{|cc|cc|}
\hline Parameter & Value & Parameter & Value \\
\hline$a_{1}$ & 1 & $b_{z 1}$ & 2 \\
$a_{4}$ & 1 & $b_{z 2}$ & 2 \\
$a_{6}$ & 1 & $k_{i 1}$ & 0.5 \\
$b_{p 1}$ & 10 & $k_{i 2}$ & 0.5 \\
$b_{p 4}$ & 10 & $k_{q 1}$ & 2 \\
$b_{p 6}$ & 10 & $k_{q 4}$ & 2 \\
$b_{q 1}$ & 2.5 & $k_{q 6}$ & 2 \\
$b_{q 4}$ & 2.5 & $k_{e 1}$ & 1 \\
$b_{q 6}$ & 2.5 & $k_{e 2}$ & 1 \\
\hline
\end{tabular}

[4] B. Roy and H. Asada, "Nonlinear feedback control of a gravityassisted underactuated manipulator with application to aircraft assembly," IEEE Transactions on Robotics, vol. 25, pp. 1125-1133, 2009.

[5] M. Ravichandran and A. Mahindrakar, "Robust stabilization of a class of underactuated mechanical systems using time scaling and lyapunov redesign," IEEE Transactions on Industrial Electronics, vol. 58, pp. 4299-4313, 2011.

[6] A. van der Schaft, L2-gain and passivity techniques in nonlinear control. Springer Verlag, 2000.

[7] R. Ortega, A. Van Der Schaft, I. Mareels, and B. Maschke, "Putting energy back in control," Control Systems, IEEE, vol. 21, pp. 18-33, 2001.

[8] R. Ortega, A. Van Der Schaft, B. Maschke, and G. Escobar, "Interconnection and damping assignment passivity-based control of portcontrolled hamiltonian systems," Automatica, vol. 38, pp. 585-596, 2002.

[9] R. Ortega, A. van der Schaft, F. Castaños, and A. Astolfi, "Control by interconnection and standard passivity-based control of porthamiltonian systems," Automatic Control, IEEE Transactions on, vol. 53, pp. 2527-2542, 2008.

[10] A. Donaire and S. Junco, "On the addition of integral action to portcontrolled hamiltonian systems," Automatica, vol. 45, pp. 1910-1916, 2009.

[11] R. Ortega and J. Romero, "Robust integral control of port-hamiltonian systems: The case of non-passive outputs with unmatched disturbancesase," Systems \& Control Letters, vol. 61, pp. 11-17, 2012.

[12] J. Romero, A. Donaire, and R. Ortega, "Robustifying energy shaping control of mechanical systems," in The 51st IEEE Conference on Decision and Control, Maui, Hawaii, USA, 2012.

[13] H. Khalil, "Nonlinear systems, 2002," Prentice Hall, Inc., 2002.

[14] C. Lanczos, The variational principles of mechanics. Dover Publications, 1986.

[15] A. van der Schaft, "Port-hamiltonian systems: an introductory survey," in International Congress of Mathematicians, 2006, pp. 1339-1365.

[16] J. Acosta, R. Ortega, A. Astolfi, and A. Mahindrakar, "Interconnection and damping assignment passivity-based control of mechanical systems with underactuation degree one," Automatic Control, IEEE Transactions on, vol. 50, pp. 1936-1955, 2005.

[17] R. Ortega, M. Spong, F. Gómez-Estern, and G. Blankenstein, "Stabilization of a class of underactuated mechanical systems via interconnection and damping assignment," Automatic Control, IEEE Transactions on, vol. 47, pp. 1218-1233, 2002.

[18] G. Viola, R. Ortega, R. Banavar, J. Acosta, and A. Astolfi, "Total energy shaping control of mechanical systems: simplifying the matching equations via coordinate changes," Automatic Control, IEEE Transactions on, vol. 52, pp. 1093-1099, 2007.

[19] R. Ortega and E. Garcia-Canseco, "Interconnection and damping assignment passivity-based control: A survey," European Journal of Control, vol. 10, pp. 432-450, 2004.

[20] C. Renton, Y. R. Teo, and T. Perez, "Total energy shaping of a class of underactuated port-hamiltonian systems using a new set of closedloop potential shape variables," in 51st IEEE Conference on Decision and Control, 2012.

21] D. Karagiannis, A. Astolfi, R. Ortega, and M. Hilairet, "A nonlinear tracking controller for voltage-fed induction motors with uncertain load torque," IEEE Transactions of Control Systems Technology, vol. 17 , pp. 608-619, 2009. 